\title{
WILLIAMS AND DOWN SYNDROMES: BRAIN ANATOMY
}

The morphological features of the cerebral hemispheres of nine subjects with Williams syndrome (WS) and six with Down syndrome (DS) have been compared using MRIs at the University of California at San Diego, La Jolla, CA. Ages ranged from 10 to 20 years (mean, 15 years). Total cerebral gray matter and total cerebral volume were reduced in both retarded groups when compared to normal controls. Regional analysis of gray matter showed significant differences between WS and DS: frontal cortex and limbic structures of the temporal lobe are disproportionately reduced while the thalamus, putamen, and globus pallidus are normal in volume in DS. Frontal and temporal limbic structures are relatively preserved in WS. (Jernigan TL, Hesselink JR et al. Cerebral morphological distinctions between Williams and Down syndromes. Arch Neurol Feb 1993; 50: 186-191). (Reprints: Dr Jernigan, Department of Psychiatry, 0631P, University of California at San Diego, 9500 Gilman Dr, La Jolla, CA 92093).

COMMENT. Previous studies in this laboratory showed cerebellar changes in these syndromes: total cerebellar size was reduced in DS and normal in WS. Regional analysis of the cerebellum of WS subjects showed a small vermis and enlarged hemispheres. The relative sparing of frontal and cerebellar structures in WS may explain the relative fluency of speech in subjects with this syndrome. Poor development of cerebellar, limbic, and frontal structures in subjects with DS correlate with their language and social-affective disabilities.

A relationship between Down syndrome and Lewy bodies, a major neuropathological feature of Parkinson's disease, is reported in two middle-aged patients examined at the Department of Neuropathology, Newcastle-upon-Tyne, UK (Raghavan R et al. Detection of Lewy bodies in trisomy 21 (Down's syndrome). Can J Neurol Sci Feb 1993; 20: 48-51). The substantia nigra was normal in size while the locus coeruleus was small. Alzheimer-type pathology was also present. An etiological connection between Parkinson's and Alzheimer's diseases and trisomy 21 was suggested.

\section{POSTERIOR FOSSA MRI IN RETARDED AUTISTIC CHILDREN}

Morphological evidence of brain stem but not cerebellar involvement in 12 autistic retarded children is reported from the University of Tokushima School of Medicine, Japan. Midsagittal MRIs showed that the brain stem was significantly smaller in the autistic group compared to 14 controls but not compared to 15 non-autistic retarded children, all groups of mean age 7 years (range, 5-10 years). The cerebellar vermis was not different among the three groups. The ratio of the midbrain to posterior fossa area was significantly 